\title{
ELECTRO-OPTIC BEAM POSITION AND PULSED POWER MONITORS FOR THE SECOND AXIS OF DARHT
}

\author{
M. A. Brubaker, C. A. Ekdahl, LANL, Los Alamos, NM 87545, USA \\ C. P. Yakymyshyn, Montana State University, MT 59717, USA
}

\begin{abstract}
The second axis of the Dual Axis Radiographic HydroTest (DARHT) facility utilizes a long pulse electron beam having a duration in excess of two microseconds. This time scale poses problems for many conventional diagnostics that rely upon electrical cables to transmit signals between the accelerator and recording equipment. Recognizing that transit time isolation is not readily achieved for the long pulse regime, difficulties resulting from ground loops are anticipated. An electro-optic (EO) voltage sensor technology has been developed to address this issue. The EO sensor exploits the Pockels effect in $\mathrm{Bi}_{4} \mathrm{Ge}_{3} \mathrm{O}_{12}$ (BGO) to provide linear modulation of laser light in response to the voltage induced on a pickup electrode. Fiber coupling between the light source, Pockels cell and receiver ensures complete galvanic isolation with improved cost and performance as compared to conventional sensors fitted with fiber optic links. Furthermore, the EO approach requires that only the passive sensor element be located near the accelerator while the light source and receiver can be installed in remote locations. This paper describes the design and development of EO sensors for electron beam and pulsed power monitoring on the second axis of DARHT. Typical calibration and testing data for the sensors is also presented.
\end{abstract}

\section{INTRODUCTION}

The first axis of DARHT provides an electron beam pulse having a full-width, half-maximum (FWHM) of 70 ns. The electron beam position and current are readily monitored using conventional B-dot probes with hardware integrators. Time isolation between the diagnostics and recording equipment is easily maintained using practical lengths of cable.

In contrast, the second axis of DARHT will provide an electron beam pulse exceeding $2 \mu$ s (FWHM) in duration. This pulse length makes accurate time integration of Bdot probes difficult and introduces the threat of ground loops since transit time isolation is no longer practical. A self-integrating electro-optic (EO) sensor offering complete galvanic isolation via optical fibers has been developed in response to these issues.

Electro-optic sensors based on the Pockels effect are gaining acceptance in the electric utility industry [1] where galvanic isolation of instrumentation is paramount. Such sensors offer advantages over conventional fiber optic links in both cost and performance. As illustrated in Fig. 1, the passive transducer element is installed on the accelerator, while the light source and receiver can be located remotely in a shielded area. EO sensors using $\mathrm{Bi}_{4} \mathrm{Ge}_{3} \mathrm{O}_{12}$ (BGO) Pockels cells have been developed for both electron beam and pulsed power monitoring on the DARHT second axis accelerator. This paper provides an overview of the sensor designs for each application along with relevant data. The theory of operation of the Pockels cell has been previously described [1-4] and is omitted for the sake of brevity.

\section{EO SENSOR PERFORMANCE}

The EO sensor is comprised of three primary components, namely a light source, sensor element and optical receiver as depicted in Fig. 1. Each component must be carefully optimized both individually and from the system perspective to obtain the best possible performance.

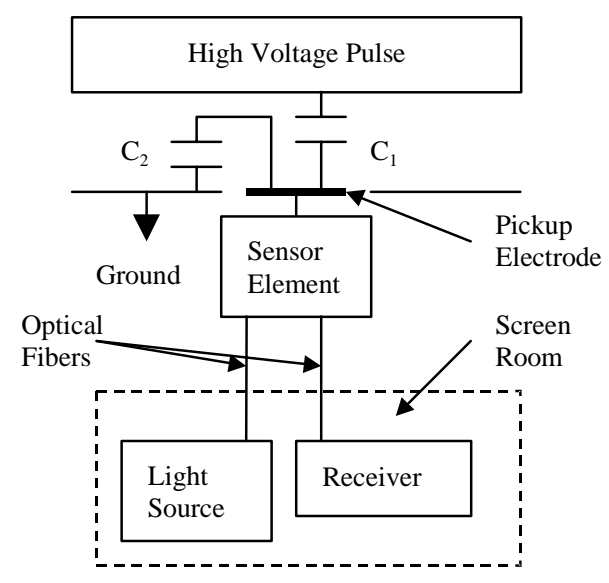

Fig. 1. EO sensor implementation.

\subsection{Goals}

The EO sensor performance goals for electron beam and pulsed power monitoring are provided in Table 1 . The former must meet the stated objectives for a current of $2 \mathrm{kA}$ in bore sizes of both 10 " (127 mm radius) and 14" (177.8 mm radius) while the latter must be achieved for a cell drive voltage of $200 \mathrm{kV}$. Note that practical limitations of existing recording instruments ultimately limit the measurement accuracy even if the sensors exceed these requirements. However, as the dynamic range of commercially available recording continues to improve, the sensor will become the limiting factor. This is particularly attractive given the bandwidth of interest for the DARHT application. 


\begin{tabular}{|l|l|}
\hline Application & Performance \\
\hline $\begin{array}{l}\text { Beam Position Monitor } \\
(\mathrm{BPM})\end{array}$ & $\begin{array}{l}\text { Position: } \pm 200 \mu \mathrm{m} \\
\text { Current: } \Delta \mathrm{I}<2 \% \\
\text { Bandwidth: } 100 \mathrm{MHz}\end{array}$ \\
\hline $\begin{array}{l}\text { Cell Voltage Monitor } \\
(\mathrm{CVM})\end{array}$ & $\begin{array}{l}\text { Flatness: } \pm 0.5 \% \\
\text { Absolute: } \pm 1 \% \\
\text { Bandwidth: } 100 \mathrm{MHz}\end{array}$ \\
\hline
\end{tabular}

Table 1. Performance goals for EO sensors.

\subsection{Critical Parameters}

For the present application, the EO sensor Pockels cell is biased at quadrature using an eighth-wave, zero-order wave plate [3]. In this case, the general response of the sensor assuming linear operation in a dual-pass configuration will be

$$
\mathrm{V}_{\text {out }}=\mathrm{GP}_{\mathrm{DC}}\left(1+2 \pi \frac{\mathrm{V}}{\mathrm{V}_{\pi}}\right)
$$

where $\mathrm{G}$ is the receiver gain factor $(\mathrm{V} / \mathrm{W}), \mathrm{P}_{\mathrm{DC}}$ is the optical power measured at the receiver (W), and $\mathrm{V}$ is the voltage induced on the Pockels cell $(\mathrm{V})$. The parameter $\mathrm{V}_{\pi}$, a measure of the Pockels cell efficiency, is given as

$$
\mathrm{V}_{\pi}=\frac{\lambda}{2 \pi \mathrm{n}_{\mathrm{o}}{ }^{3} \mathrm{r}_{41}}
$$

where $\lambda$ is the optical wavelength $(m), n_{o}$ is the bulk refractive index and $r_{41}$ is the Pockels coefficient $(\mathrm{m} / \mathrm{V})$.

The EO sensor acts as a capacitive divider with a voltage induced upon the pickup electrode in response to the high voltage source of interest as shown in Fig. 1. The magnitude of the induced voltage is dictated by the ratio $\mathrm{C}_{1} / \mathrm{C}_{2}$ for $\mathrm{C}_{1} \ll \mathrm{C}_{2}$. This ratio can be readily optimized for each application to ensure that the voltage is sufficient to provide adequate resolution without violating the criteria for linear operation $\left(\mathrm{V} \ll \mathrm{V}_{\pi}\right)$. For BGO, $\mathrm{V}_{\pi}$ is on the order of $46 \mathrm{kV}$ and $71 \mathrm{kV}$ at respective operating wavelengths of $850 \mathrm{~nm}$ and $1319 \mathrm{nmm}$.

The signal-to-noise ratio for the EO sensor is approximated using the second term of Eq. 1 in divided by the combination of various noise sources as

$$
\frac{\mathrm{S}}{\mathrm{N}}=\frac{\mathrm{P}_{\mathrm{DC}} \mathrm{G}\left(2 \pi \frac{\mathrm{V}}{\mathrm{V}_{\pi}}\right)}{\sqrt{\left(\mathrm{P}_{\text {shotnoise }}\right)^{2}+\left(\mathrm{P}_{\text {receivernoise }}\right)^{2}+\left(\mathrm{P}_{\text {lasernoise }}\right)^{2}}} .
$$

Note that the noise terms are defined as root-mean-square (rms) values. The first term in the denominator represents the shot noise equivalent power for the photo-detector and provides an indication of the theoretical limit, assuming that classical light is used. The remaining noise terms must be minimized by proper selection of the light source and receiver. Having defined the limiting parameters for the EO sensor, the optimization of the light source, sensor element and receiver may now be addressed.

\subsection{Light Source}

The ideal light source for the EO sensor provides high optical power ( $>5 \mathrm{~mW}$ ) with very low relative intensity noise (RIN $<-160 \mathrm{~dB} / \mathrm{Hz}$ ) to permit shot noise limited performance. Various commercial Fabry-Perot laser diodes offer high power well above $100 \mathrm{~mW}$, but are quickly rejected due to poor noise performance. At 850 $\mathrm{nm}$, vertical cavity surface emitting lasers (VCSEL's) provide significantly better noise characteristics (RIN < $125 \mathrm{~dB} / \mathrm{Hz}$ ) at up to $10 \mathrm{~mW}$, yet the noise performance is still not adequate. Light emitting diodes (LED's) offer excellent noise characteristics at $850 \mathrm{~nm}$, but suffer from insufficient power levels $(<1 \mathrm{~mW})$.

At $1319 \mathrm{~nm}$, distributed feedback (DFB) lasers provide acceptable power levels and noise performance, but are difficult to stabilize for consistently low noise levels. Edge emitting LED's at this wavelength do not provide sufficient optical power and operate well above the shot noise limit. The most attractive option at $1319 \mathrm{~nm}$ is a diode-pumped Nd:YAG non-planar ring (NPRO) laser which offers very high power (> $200 \mathrm{~mW}$ ) with extremely low noise levels (RIN $<-160 \mathrm{~dB} / \mathrm{Hz}$ ).

The choice of light source (and operating wavelength) is thus narrowed to the $850 \mathrm{~nm}$ VCSEL and the $1319 \mathrm{~nm}$ YAG laser. The latter device was ultimately selected for superior noise performance and completely eliminates the laser noise term from the denominator of Eq. 3. A single laser can be fanned-out using fiber optic couplers to drive multiple sensors and the amortized cost is actually comparable to using one VCSEL per sensor. However, a very narrow laser line width leads to interferometric effects throughout the system. The narrow line width gives an extremely long coherence length that makes it difficult to depolarize the highly polarized output of this laser source. Variations in the polarization state at each sensor cause unwanted intensity fluctuations.

\subsection{Sensor Element}

A common Pockels cell design illustrated in Fig. 2 is used for both monitoring applications. The physical scale may be considered in terms of the BGO crystal, which is $10 \mathrm{~mm}$ long and $3.5 \mathrm{~mm}$ in diameter. The details of each specific sensor envelope are addressed in later sections.

A borosilicate window serves as the substrate for mounting the BGO crystal and external optics. The BGO crystals have anti-reflective (AR) and highly-reflective (HR) coatings applied at opposite ends. The former coating is necessary to minimize back reflections in the system while the latter provides the mirror required for dual-pass operation. The AR-coated end of the BGO is bonded to the window substrate using epoxy and a silver epoxy electrode is applied to the HR-coated end. The entire BGO crystal is then potted in a composite of epoxy 


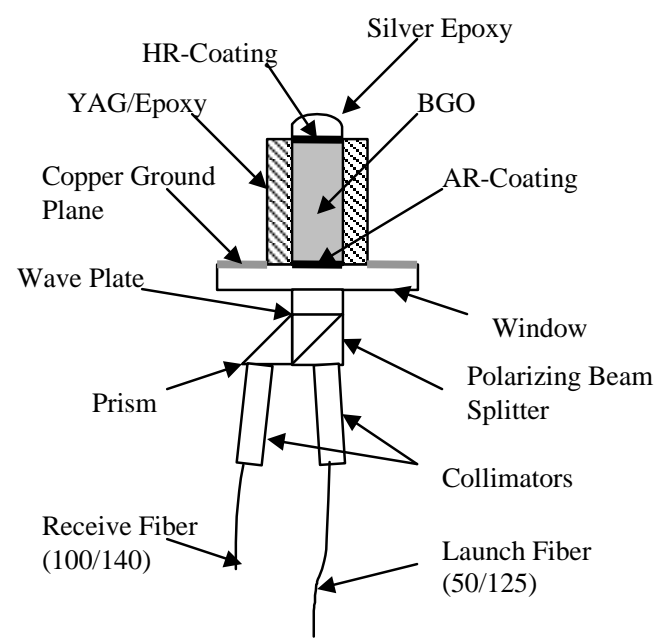

Fig. 2. EO sensor Pockels cell design.

and yttrium aluminum garnet (YAG) powder, which secures a copper sheet ground plane to the window surface.

The potting is required to combat a weak photo-elastic effect in the BGO crystal that creates unwanted strain birefringence when an electric field is applied [5]. The YAG/epoxy blend is selected to provide an acoustically lossy medium that matches the acoustic impedance BGO. This approach substantially reduces the acoustic error of the EO sensor, which varies with the spectral content of the applied field and can be problematic for the time scale of interest.

The required eighth-wave wave plate and polarizing beam splitter cube are bonded to the other side of the window after proper rotational alignment to the BGO crystal. Collimators using quarter-pitch graded index (GRIN) lenses couple light between the sensor and input and output multi-mode optical fibers. A slight splaying of the collimators combined with the AR-coating minimize interferometric effects in the sensor element, which cause calibration variations with temperature and wavelength. Typical sensor insertion losses are $-9 \mathrm{~dB}$, which is quite acceptable recognizing that $-6 \mathrm{~dB}$ is the theoretical limit accounting for only the polarizer attenuation.

\section{4 Receiver}

An optical receiver was developed in-house to meet the EO sensor requirements. Shot noise limited performance has been obtained up to $100 \mathrm{MHz}$. The production receiver utilizes surface mount technology and incorporates an automatic gain control to correct for changes in the optical received power to maintain the correct calibration.

\section{BEAM POSITION MONITOR}

\subsection{Housing Design}

The DARHT second axis accelerator has very strict vacuum requirements and all sensors will be checked to a maximum allowable leak rate of $1.7 \times 10^{-8}$ Torr/liters/second. To achieve this specification, the EO sensor has a stainless steel vacuum housing (metal seal to beam line) and a brazed alumina feed-through for the pickup electrode as shown in Fig. 3. A spring-loaded pin is utilized to ensure a good electrical contact to the Pockels cell electrode. The Pockels cell window is captured by a counter-bored flange and held in place with nylon-tipped set screws. Excess fiber from the collimator pigtails is coiled in the air-side housing and bulkhead FC mating sleeves facilitate external connections. All of the fiber optic connectors are angle polished to eliminate interferometric effects in the cabling. Finally, an external bias connection to the Pockels cell electrode is also provided. Up to $1 \mathrm{kV}$ DC can be applied to the electrode through a $500 \mathrm{M} \Omega$ resistor. The bias connection also provides a means of performing an in situ calibration of the EO sensor with a fast pulse source and reference.

\subsection{Calibration Results}

Beam position monitors using four equally spaced $\mathrm{EO}$ sensors have been calibrated using the DARHT second axis calibration stand [6]. After modifying the EO sensor design to eliminate interferometry errors, the calibration factor stability typically indicates a standard deviation of $1 \%$. A representative measurement of the test line current waveform by an EO BPM in a 10" (127 mm radius) bore is compared against the reference current

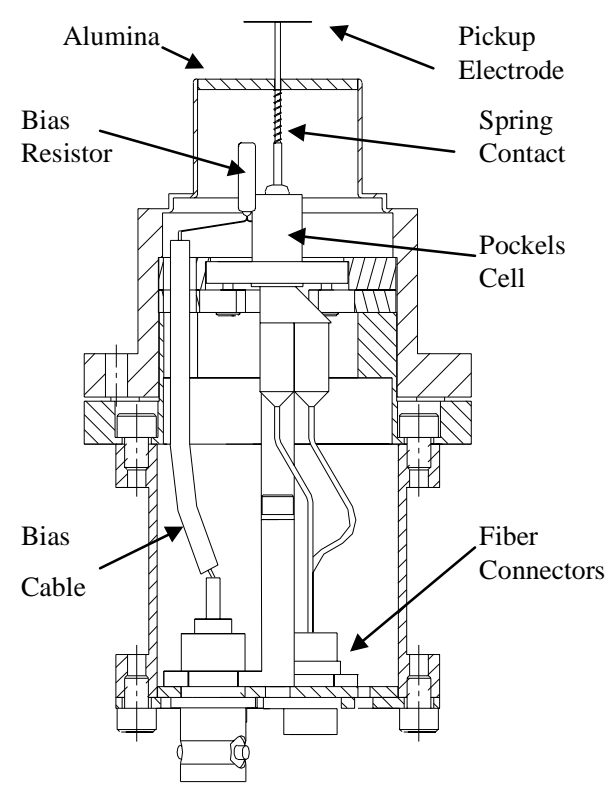

Fig. 3. EO sensor housing for beam position monitoring. 


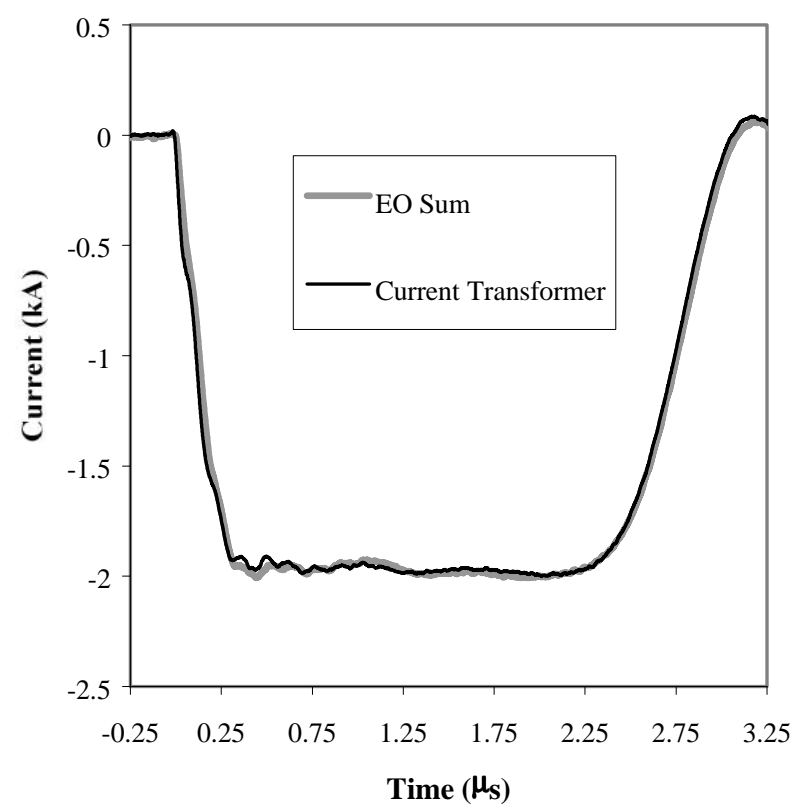

Fig. 4. EO sensor and reference current transformer waveform comparison on calibration stand.

transformer in Fig. 4. Good agreement is obtained, but a small acoustic error (less than 2\%) is still present in the EO sensor response. The induced voltage on the EO sensor electrodes will be on the order of $1.5 \mathrm{kV}$ for this configuration, which satisfies the criterion for linear operation.

For small displacements of the center conductor, the acoustic effect is common mode and the EO sensor array calculates the position to better than \pm 500 microns as shown in Fig. 5. The position is calculated as the difference between opposite detectors normalized by the sum of the sensor outputs and multiplied by the pipe radius. Note that the effect of the non-zero radius of the inner conductor on the current centroid position has been corrected for in the analysis. The relative accuracy is found to be consistently better than $\pm 300 \mu \mathrm{m}$ over the flat top of the pulse. All of this data was taken with seven effective bits

\section{CELL VOLTAGE MONITOR}

\subsection{Housing Design}

The EO cell voltage monitor is mounted on an external compensation can where the cell ballast resistors are located. The sensor electrode observes the drive rod voltage through a polycarbonate nose having the same dielectric constant as the insulating oil. An O-ring fits in the grooved portion of the polycarbonate to form a piston seal with the diagnostic port. As illustrated in Fig. 6, the Pockels cell window is secured in a counter bore using a threaded ring. A contact spring connects the Pockels cell and pickup electrode. Similar to the beam position sensor, the excess fiber is stored in an external can fitted with a connector plate. All fiber optic connectors are

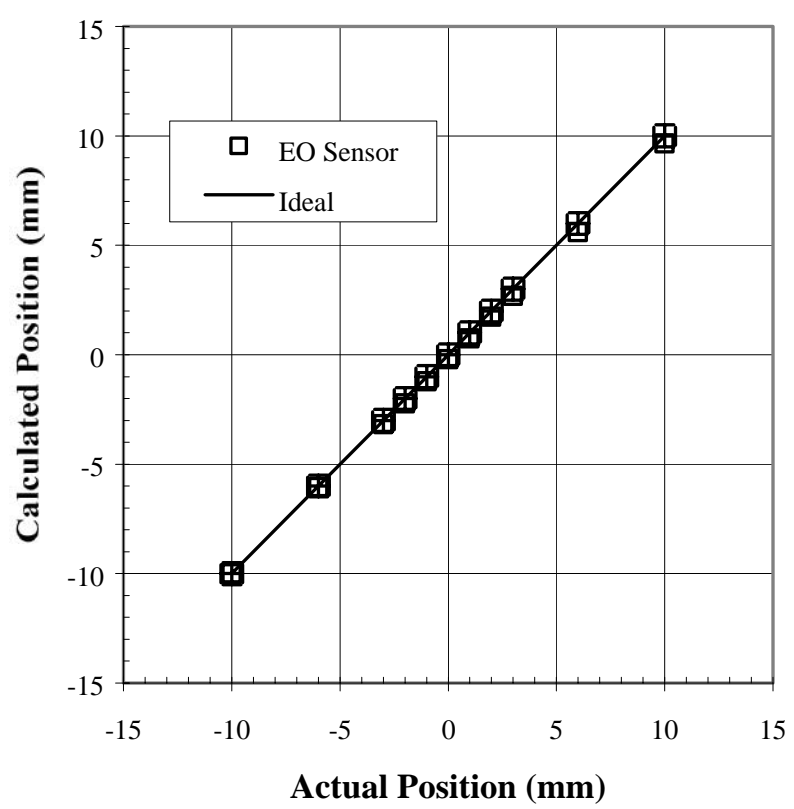

Fig. 5. Computed versus actual conductor offset for $\mathrm{EO}$ sensor beam position monitor.

angle polished to prevent back reflections that can cause interferometric effects in the fiber runs.

\subsection{Preliminary Results}

Testing of EO cell voltage monitors is currently in progress at LANL using the accelerator cell production test stand. At nominal voltage $(200 \mathrm{kV})$ the EO sensor pickup electrode is expected to reach an induced voltage of $3 \mathrm{kV}$, which is still within the linear range of the sensor. Initial results indicate some disagreement between the EO sensor and compensation can resistive divider. Typical data at less than half of nominal voltage are presented in Fig. 7, which shows that the EO sensor predicts a faster front time. At higher voltages, the discrepancy becomes worse, approaching $10 \%$ at $200 \mathrm{kV}$. Possible explanations are a voltage dependence of the resistive divider and acoustic error in the EO sensor. Efforts are currently under way to resolve this issue.

\section{CONCLUSIONS}

The results presented here indicate that the BPM sensors are approaching the goals specified in Table 1 . The relative accuracy of the beam position measurement is on the order of \pm 300 microns and is ultimately limited by the noise floor and acoustic differences between sensors. Filtering out the acoustic signature may reduce the latter effect. The absolute accuracy is currently at \pm 500 microns and the fidelity of the current measurement is within $2 \%$. The limiting factor for absolute position accuracy is believed to be offset errors in the receiver gain control. This effect is aggravated by residual polarization variations at the sensor, which can cause significant intensity fluctuations at the receiver, depending on the orientation of the principle polarization axes to the sensor polarizers. Work is in progress to address this problem 


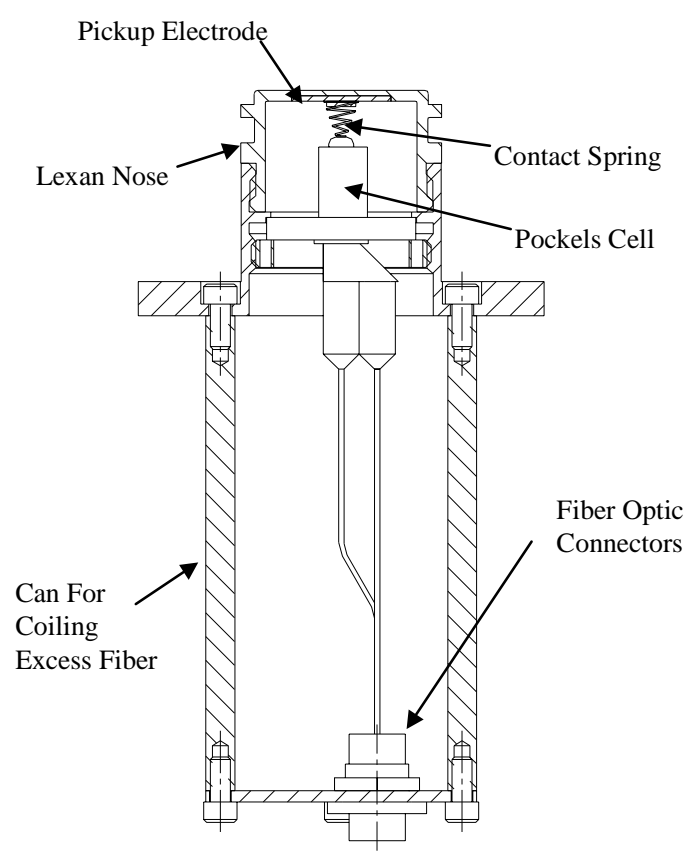

Fig. 6. EO sensor design for cell voltage monitoring application.

through high-speed modulation of the residual polarization.

The EO cell voltage monitor can provide the absolute accuracy specified in Table 1, but satisfaction of the flatness requirement cannot yet be proven. Work is in progress to resolve the discrepancies between the EO sensor and resistive divider pulse shapes. Capacitive Edot probes may ultimately provide a better confirmation of the drive rod voltage subject to integration difficulties for this time scale. Acoustic error is expected to be the ultimate limitation for the EO sensor in this case. Recognizing that the acoustic mode exhibits a high Q resonant frequency, the use of post-processing filtering should provide improved results.

\section{ACKNOWLEDGEMENTS}

The authors gratefully acknowledge the contributions of Anthony Chavez, Steve Eversole, Melissa Reed, Patrick Rodriguez and Wayne Smith of LANL. In addition, the efforts of Bill Broste, Doug Devore, Bruce Marshall and Jeff Johnson of Bechtel Nevada are highly appreciated. The work undertaken at MSU by students Adam Iverson and Irene Vandeveegaete is gratefully acknowledged. Finally, we thank Dr. James Downing of LANL for his continued support of this work and numerous technical inputs. This work was funded by the U. S. Department of Energy under contract W-7405-Eng-36. Work at MSU was partly funded by the DOE EPSCOR Lab Partnerships Program under contract DE-FG02-98ER45720.

\section{REFERENCES}

[1] Brubaker, M. A., Johnston, P. M., Reinbold, C, and Yakymyshyn, C. P,"Manufacturing Challenges of Optical Current and Voltage Sensors for Utility Applications," Proceedings of SPIE, Volume 3201, pp 1-19.

[2] Brubaker, M.A. and Yakymyshyn, C. P.,"An ElectroOptic Voltage Sensor for Electron Beam Position and Current Monitoring," IEEE Transactions on Plasma Science, Vol. 28, No. 5, October 2000.

[3] Brubaker, M. A., Iverson, A. J., Vandeveegaete, I. E. and Yakymyshyn, C. P., "A Prototype Electro-Optic Voltage Sensor for Accelerator Diagnostic Application, Proceedings of the $12^{\text {th }}$ IEEE International Pulsed Power Conference, Monterey, CA, 1999, pp 619-622.

[4] Brubaker, M. A. and Yakymyshyn, C. P.,"Pockels Cell Voltage Probe for Non-Invasive Electron Beam Measurements," Applied Optics, Vol. 39, No. 7, March 2000, pp 1159-1163.

[5] Yariv, A. and Yeh, P., Optical Waves in Crystals, Wiley, New York, 1984.

[6] Carlson, R. L. , "A 100-kV, 2-kA, $2.5 \mu$ s Pulser for Developing and Calibrating Long-Pulse Diagnostics," Proceedings of the $12^{\text {th }}$ IEEE International Pulsed Power Conference, Monterey, CA, 1999, pp 1429-1432.

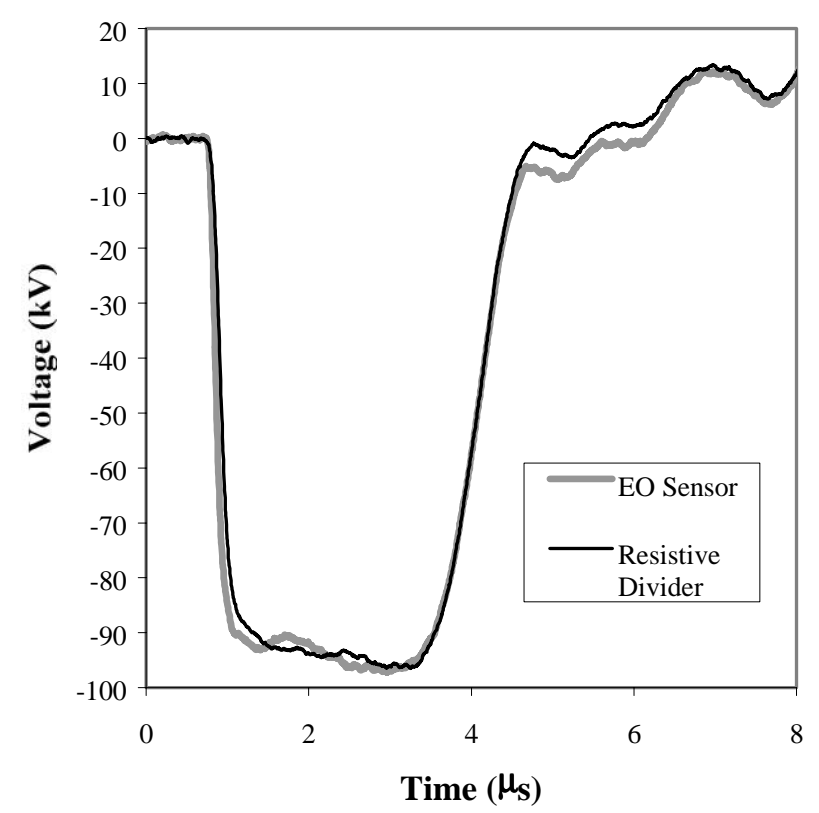

Fig. 7. Comparison of EO cell voltage monitor and resistive divider. 